\title{
Zeeman Splitting in the Diffuse ISM
}

\author{
Carl Heiles \\ Astronomy Department, University of California, Berkeley CA 94720-3422, USA \\ email: heiles@astro.berkeley.edu
}

\begin{abstract}
Many Zeeman-spitting measurements in the diffuse Galactic Interstellar Medium have been made of the 21-cm line in both absorption and emission. Typical field strength is about $6 \mu \mathrm{G}$, with enhancement in shocked regions; the magnetic, turbulent, and cosmic-ray pressures are comparable and considerably larger than the thermal gas pressure. For PhotoDissociation Regions, Carbon recombination lines show intriguing results for single-dish measurements. $\mathrm{OH}$ Megamasers in Ultra-Luminous Infrared Galaxies show easily detectable fields whose strength ranges up to at least $20 \mathrm{mG}$. Upper limits for several damped Ly- $\alpha$ systems range down to a few $\mu \mathrm{G}$. The $\mathrm{z}=0.692$ system against $3 \mathrm{C} 286$ was reported in the literature to have a large field strength, but this result is wrong.
\end{abstract}

Keywords. ISM: magnetic fields, galaxies: magnetic fields, masers, polarization

\section{Historical Introduction}

Galactic ISM Studies in the 21-cm line. Zeeman splitting of the Interstellar Medium (ISM) was discovered for the 21-cm line in absorption against the powerful continuum source Cas A, and a few other sources, by Verschuur (1969). No further work was done in absorption for more than thirty years, when Heiles and Troland (a series culminating in 2005) surveyed some 70 sources using the Arecibo telescope for their "Millennium Survey". Meanwhile, work concentrated on mapping the field using the $21-\mathrm{cm}$ line in emission. This resulted in a catalog of 429 positions (Heiles, 1988; Heiles, 1989; Heiles, 1994; Myers et al. 1995) observed with the Hat Creek 85-foot telescope (which was destroyed by a windstorm in winter 1993), plus a few more by Verschuur (1989). Meanwhile, Verschuur (1995a, 1995b) severely criticized all this work, claiming that all emission results were spurious owing to polarized sidelobes. This criticism was put to rest by analysis of the North Celestial Pole data (Heiles 1996), the analysis of his criticism by Heiles (1998), and the observations and analyses by Robishaw (2008).

Nevertheless, polarized beam structure plagues measurements of Zeeman splitting in 21-cm line emission, even for the Green Bank Telescope with its clear aperture and supposedly minimal sidelobes (Robishaw and Heiles 2009).

Galactic and Extragalactic Studies of Small Sources. Polarized sidelobes are irrelevant for small sources, including $\mathrm{OH}$ masers and star forming regions (see Crutcher, this conference). Observations have been performed of three additional classes of small source: PhotoDissociation Regions (PDRs; often called Photon-Dominated Regions), which are the highly compressed regions lying between HII regions and the un-ionized ambient medium; OH Megamasers (OHMs) at moderate redshifts in Ultra-Luminous Infrared Galaxies (ULIRGs); and Damped Lyman- $\alpha$ Absorption (DLA) line systems. PDRs yield intriguing results that justify the time and effort involved in high-resolution mapping of the Zeeman splitting, for example with the VLA. OHMs have turned into a field with many interesting and strong results, currently being pursued with vigor. DLAs have only negative results, despite a recent report of an amazingly strong field towards 3C286 (Wolfe et al. 2008) which has subsequently been shown to be wrong (see below).

\section{Some Details and Results}

Magnetism is a Major Force in the Diffuse ISM. The Millennium Survey of Zeeman splitting of the 21-cm line in absorption showed that the Cold Neutral Medium has a well-defined median field strength of about $6 \mu \mathrm{G}$ (Heiles \& Troland 2005). This makes it comparable to the volumeaverage Galactic field strength (Ferrière 2001) and means it dominates the gas thermal pressure 
by a factor of typically about 3 and is comparable to the turbulent pressure and the cosmic-ray pressure. The emission results are consistent with this, and in particular show field amplification in morphologically interesting regions such as shocks (Heiles 1989).

Zeeman Splitting is Probably Mappable in some PDRs. In his thesis, Robishaw has Zeeman splitting results on the Carbon Recombination Line emission in several regions, obtained with the GBT. The formal significance on the results is roughly $3 \sigma$, which is barely adequate for a single source, but because 4 out or 5 sources have this level of significance the probable reality of significant detections is enhanced. The regions include DR21 $(920 \pm 310 \mu \mathrm{G})$, Orion $(290 \pm 150$ $\mu \mathrm{G})$, M17 $(2530 \pm 860 \mu \mathrm{G})$, and DR21OH $(4940 \pm 2500 \mu \mathrm{G})$; the only source examined with a formally insignificant detection was $\mathrm{W} 3$, with $(60 \pm 110 \mu \mathrm{G})$, but this might simply reflect unfavorable viewing geometry.

Zeeman Splitting of OHMs in ULIRGS. OH Megamaseers are often easy to detect, at least at Arecibo; similarly, their Stokes V signals, which reveal Zeeman splitting, are often strong and easy to detect. Robishaw (2008) presents a pilot study of 6 sources, which have fields ranging up to about $20 \mathrm{mG}$. We are currently surveying about $70 \mathrm{OHMs}$ - the known list in Arecibo's declination range of -1 to $39 \mathrm{deg}$ - and have many detections.

Zeeman Splitting in Damped Ly- $\alpha$ systems. Lane \& Heiles (in preparation) observed and found upper limits in 3 DLAs in 2 sources, $0738+313(1.8 \pm 2.4 \mu \mathrm{G}$ for $\mathrm{z}=0.0912 ; 12.5 \pm$ $9.0 \mu \mathrm{G}$ for $\mathrm{z}=0.2212)$ and $0235+164(\lesssim 10 \mu \mathrm{G}, \mathrm{z}=0.524)$. Wolfe et al. (2008) observed 3C286 and reported the amazing $84 \pm 9 \mu \mathrm{G}$. It turns out, however, that this result is wrong. Owing to a software bug, a phase angle was not accounted for. This angle happened to be about $90^{\circ}$, which meant that the purported circular polarization is really linear. The absorption line is strongly linearly polarized and is of interest in itself. However, the magnetic field limit is $(12.7 \pm 5.8) \mu \mathrm{G}$, an insignificant result.

\section{References}

Ferrière, K. 2001, RevModPhyss, 73, 1031

Heiles, C. 1988, ApJ, 324, 321

Heiles, C. 1989, ApJ, 336, 808

Heiles, C. 1994, ApJ, 424, 208

Heiles, C. 1996, ApJ 466, 224

Heiles, C. 1997, ApJS 111, 245

Heiles, C. 1998, Astrophys. Lett. and Comm. 37, 85

Heiles, C. \& Troland, T. H./ 2005, ApJ 624, 773

Myers, P. C., Goodman, A. A., Rüsten, R., \& Heiles, C. 1995, ApJ, 442, 177

Robishaw, T. 2008, Ph.D. Thesis, University of California, Berkeley (AAT 3331778)

Robishaw, T. \& Heiles, C. 2009, PASP, 121, 272

Robishaw, T., Quataert, E., \& Heiles, C. 2008, ApJ, 680, 981

Verschuur, G. L. 1969, ApJ, 156, 861

Verschuur, G. L. 1989, ApJ, 339, 163

Verschuur, G. L. 1995a, ApJ, 451, 624

Verschuur, G. L. 1995b, ApJ, 451, 645

Wolfe, A. M., Jorgenson, R. A., Robishaw, T., Heiles, C., \& Prochaska, J. X. 2008, Nature, 455, 638 